\title{
FINE TOPOLOGY ON FUNCTION SPACES
}

\author{
R.A. McCOY \\ Department of Mathematics \\ Virginia Polytechnic Institute and State University \\ Blacksburg, Virginia 24061 U.S.A.
}

(Received December 23, 1985 and in revised form January 28, 1986)

ABSTRACT. This paper studies the topological properties of two kinds of "fine topologies" on the space $\mathrm{C}(\mathrm{X}, \mathrm{Y})$ of all continuous functions from $\mathrm{X}$ into $\mathrm{Y}$. KEY WORDS AND PHRASES. Function spaces, uniform topology, fine uniform topology. 1980 MATHEMATICAL SUBJECT CLASSIFICATION CODE. $54 \mathrm{C} 35$.

\section{INTRODUCTION.}

The topology of pointwise convergence and the comnact-onen topology are two of the most commonly used topologies on the set $\mathrm{C}(\mathrm{X}, \mathrm{Y})$ of continuous functions from a space $X$ into a space $Y$. These spaces will be denoted by $C_{D}(X, Y)$ and $\mathrm{C}_{\mathrm{y}}(\mathrm{X}, \mathrm{Y})$, respectively. If $\mathrm{Y}$ is a metric space, the supremum metric topology on $\mathrm{C}(\mathrm{X}, \mathrm{Y})$ is also commonly used. However, sometimes none of these topologies is strong enough to apply a function space to a given situation, in which case a finer topology may be needed. A good example of this is the use of a "fine topology" on a function space in [4], in which the Baire space property of the function space is used to obtain certain kinds of embeddings into infinite-dimensional manifolds.

This paper studies the topological properties of two kinds of "fine topologies" on $\mathrm{C}(\mathrm{X}, \mathrm{Y})$. In order to avoid pathologies, all spaces will be Tychonoff spaces. The symbol $\mathbb{R}$ will denote the real line with the usual topology, and $\mathbb{R}^{+}$will denote the positive real line. Also $C(X, \mathbb{R})$ and $C\left(X, \mathbb{R}^{+}\right)$will be abbreviated as $C(X)$ and $\mathrm{C}^{+}(\mathrm{X})$. Finally let $\omega$ denote the set of natural numbers.

1. UNIFORM TOPOLOGIES.

Whenever a space $Y$ has a compatible uniform structure on it, this induces a uniform structure on $\mathrm{C}(\mathrm{X}, \mathrm{Y})$. If $\delta$ is a diagonal uniformity on $\mathrm{Y}$, then for each

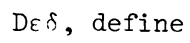

$$
\hat{D}=\left\{(f, g) \varepsilon C(X, Y)^{2} \text { : for every } x \varepsilon X,(i(x), g(x)) \varepsilon D\right\} \text {. }
$$

The family $\{\hat{D}: D \varepsilon \delta\}$ is a base for a diagonal uniformity $\hat{\delta}$ on $C(X, Y)$. Denote the resulting topological space by $C_{\delta}(X, Y)$. On the other hand, if $\mu$ is a covering uniformity on $Y$, then for each $U_{\varepsilon \mu}$, let

$\hat{u}=\left\{(f, g) \varepsilon C(X, Y)^{2}\right.$ : for every $x \in X$, there exists a $U \varepsilon U$ with $\left.(f(x), g(x))=U^{2}\right\}$.

The family $\left\{\hat{u}: U_{\varepsilon \mu}\right\}$ is also a base for a diagonal uniformity $\hat{\mu}$ on $C(X, Y)$. Denote this space by $C_{\mu}(X, Y)$. 
There is a natural way of passing from a diagonal uniformity $\delta$ to a covering uniformity $\mu_{\delta}$, so that $\mu_{\delta_{\mu}}=\mu$ and $\delta_{\mu_{\delta}}=\delta$ (cf. Willard [5], section 36). It can be easily verified that $C_{\mu \delta}(X, Y)=C_{\delta}(X, Y)$ and $C_{\delta}(X, Y)=C_{\mu}(X, Y)$. Therefore a uniform structure on $Y$ may be considered either as a diagonal uniformity or a covering uniformity, and the resulting uniform structures on $\mathrm{C}(\mathrm{X}, \mathrm{Y})$ will generate the same topology, called the uniform topology.

Let $v$ stand for the fine (covering) uniformity on $Y$. Whenever $Y$ is paracompact, $\checkmark$ has as a base the family of all open covers of $Y$. The topology on $C_{v}(X, Y)$ will be called the fine uniform topology.

If $\mu$ is any compatible uniformity on $Y$, then the relationships between the various topologies discussed above are given by

$$
C_{p}(X, Y) \leq C_{k}(X, Y) \leq C_{\mu}(X, Y) \leq C_{v}(X, Y),
$$

where the inequality means the space on the right is finer than that on the left.

Each compatible bounded metric $\rho$ on $\mathrm{Y}$ induces the supremum metric $\hat{\rho}$ on $\mathrm{C}(\mathrm{X}, \mathrm{Y})$, defined by $\hat{\rho}(f, g)=\sup \{\rho(f(x), g(x)): x \in X\}$. The resulting topological space will be depoted by $C_{\rho}(X, Y)$. A base for $C_{\rho}(X, Y)$ consists of the metric balls $\left\{B_{\rho}(f, e): f \in C(X, Y)\right.$ and $\left.e \varepsilon \mathbb{R}^{+}\right\}$. If $\mu_{\rho}$ is the uniformity on $Y$ generated by $\rho$, then $\mathrm{C}_{\mu_{\rho}}(\mathrm{X}, \mathrm{Y})=\mathrm{C}_{\rho}(\mathrm{X}, \mathrm{Y})$.

For a metrizable space $Y$, let $M(Y)$ be the family of all compatible bounded metrics on $Y$. The following fact gives a useful tool for working with the fine uniform topology.

PROPOSITION 1.1. If $Y$ is metrizable, then $C_{V}(X, Y)$ has as a base $\left\{B_{\rho}(f, e): \rho \varepsilon M(Y), f \varepsilon C(X, Y)\right.$ and $\left.e \varepsilon \mathbb{R}^{+}\right\}$.

PROOF. To see that $B_{\rho}(f, e)$ is a neighborhood of $f$ in $C_{v}(X, Y)$, define the open cover $U=\left\{B_{\rho}(y, e / 3): y \varepsilon Y\right\}$ of $Y$, and let $g \varepsilon l[f]$. Then for each $x \varepsilon X$, there is a yєY with $(f(x), g(x)) \in B_{p}(y, e / 3)$. Therefore each $\rho(f(x), g(x))<2 e / 3$, so that $\hat{\rho}(f, g) \leq 2 e / 3<e$. This establishes that $g \varepsilon B_{\rho}(f, e)$, and $i t$ follows that $\hat{U}[f] \subset B_{\rho}(f, e)$. On the hand, let $U_{\varepsilon \mu}$ and $f \in C(X, Y)$. Let $U_{1}>^{*} U_{2}>^{*} \ldots$ be a normal sequence of open covers of $Y$ so that $U_{1}$ refines $U$, and let $\rho$ be the metric defined by this sequence (see Willard [5], p. 167, for this construction). It follows from the construction of $\rho$ that there is an $e \in \mathbb{R}^{+}$so that $B_{\rho}(f, e) \subset \hat{u}[f]$.

Let $\mu$ be any compatible uniformity on $Y$. If $X$ is compact then $\hat{\mu}$ on $C(X, Y)$ is the same as the uniformity of uniform convergence on comnact sets, which is known to generate the compact-open topology (cf. Willard [5], section 43). The converse is in fact also true for most $Y$.

PROPOSITION 1.2. If $Y$ contains a nontrivial path, then for any compatible uniformity $\mu$ on $Y, C_{\mu}(X, Y)=C_{K}(X, Y)$ if and only if $X$ is compact.

PROOF. Let $\phi: I \rightarrow Y$ be a continuous function from the closed unit interval into $Y$ such that $\phi(0) \neq \phi(I)$. Let $y=\phi^{\prime}(0)$, let $z=\phi(1)$, and let $f$ be the constant man from

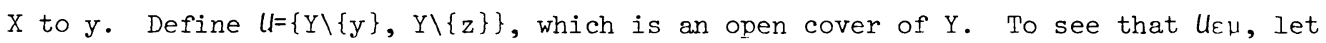
$D \varepsilon \delta_{\mu}$ with $z \notin D[y]$. Choose a symmetric $E \varepsilon \delta_{\mu}$ with $E O E \subset D$. It follows that $\{E[p]: p \varepsilon Y\}$ refines $U$, so that $U_{\varepsilon \mu}$. 
Suppose that $X$ is not compact. To see that $\hat{U}[f]$ is not a neighborhood of $f$ in $C_{k}(X, Y)$, let $W=\left[A_{1}, V_{l}\right] \cap \ldots \cap\left[A_{n}, V_{n}\right]$ be any basic open subset of $C_{k}(X, Y)$ which contains $f$, where each $\left[A_{i}, V_{i}\right]=\left\{f \varepsilon C(X, Y): f\left(A_{i}\right) \subset V_{i}\right\}$ and the $A_{i}$ are compact and the $V_{i}$ are open. If $A=A_{1} \cup \ldots \cup A_{n}$, then there is some $x \varepsilon X \backslash A$. Define $g: A \cup\{x\} \rightarrow Y$ by $g(a)=y$ for aEA and $g(x)=z$. Since $\phi(I)$ is arcwise connected, then $g$ extends to some $\hat{g} \varepsilon C(X, Y)$. Then $\hat{g} \varepsilon W \backslash \hat{U}[f]$, which establishes that $C_{\mu}(X, Y)$ is finer than $C_{k}(X, Y)$.

It follows from Proposition 1.2 that whenever $X$ is compact, all compatible uniformities on $Y$ generate the same topology on $C(X, Y)$ - the compact-open topology. Also when $Y$ is compact, a compatible uniformity on $Y$ generates a unique topology on $C(X, Y)$ since there is only one compatible uniformity on $Y$ - but in this case the topology on $\mathrm{C}(\mathrm{X}, \mathrm{Y})$ is not the compact-open topology, unless $\mathrm{X}$ is also compact.

PROPOSITION 1.3. Let $Y$ be a metrizable space. If $X$ is psuedocompact, then all compatible uniformities on $Y$ generate the same topology on $\mathrm{C}(\mathrm{X}, \mathrm{Y})$.

PROOF. Let $U$ be an open cover of $\mathbb{R}$, and let $f \varepsilon C(X, Y)$. For each $x \in X$, let $V_{x} \varepsilon U$ with $f(x) \varepsilon V_{x}$. Then for each such $x$ there exists a $U_{x} \varepsilon \mu$ with $\left(D_{x} \circ D_{x}\right)[f(x)] \subset V_{x}$, where $D_{x}=U\left\{U^{2}: U \varepsilon U_{x}\right\}$. Since $X$ is psuedocompact and $Y$ is metrizable, then $f(x)$ is compact. So there exist $x_{1}, \ldots, x_{n} \varepsilon x$ such that $f(x) \subset D_{x_{1}}\left[f\left(x_{1}\right)\right] \cup \ldots u D_{x_{n}}\left[f\left(x_{n}\right)\right]$. Now there exists an $u_{0} \varepsilon \mu$ which refines each of $u_{x_{1}}, \ldots, u_{x_{n}}$.

In order to show that $\hat{U}_{0}[f] \subset \hat{U}[f]$, let $g \varepsilon \hat{U}_{0}[f]$ and $x \in X$. Then there exists an $i$ such that $f(x) \varepsilon D_{x_{1}}\left[f\left(x_{i}\right)\right]$. Let $U \varepsilon U_{0}$ with $(f(x), g(x)) \varepsilon U^{2}$. There is some $U_{i} \varepsilon U_{x_{I}}$ such that $U \subset U_{i}$, so that $(f(x), g(x)) \varepsilon U_{i}^{2} \subset D_{x_{j}}$. Therefore $f(x) \in D_{x_{1}}\left[f\left(x_{i}\right)\right] \subset\left(D_{x_{1}} \circ D_{x_{1}}\right)\left[f\left(x_{i}\right)\right] \subset v_{x_{I}}$ and $g(x) \varepsilon\left(D_{x_{1}} \circ D_{x_{1}}\right)\left[f\left(x_{i}\right)\right] \subset v_{x_{1}}$. Since $x$ is

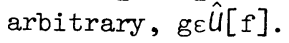

The topology generated in Proposition 1.3 is in general not the compact-open topology.

The "completeness" of a function space can be a useful property for obtaining the existence of certain kinds of functions. If $\rho$ is a complete metric on $Y$, then $\hat{\rho}$ is a comnlete metric on $\mathrm{C}(\mathrm{X}, \mathrm{Y})$. On the other hand, $\mathrm{C}_{v}(\mathrm{X}, \mathrm{Y})$ may not be metrizable. So complete metrizability of $\mathrm{C}_{V}(\mathrm{X}, \mathrm{Y})$ is too much to expect in general. $\mathrm{But} \mathrm{C}_{\nu}(\mathrm{X}, \mathrm{Y})$ does have "completeness" to the following extent.

THEOREM 1.4. If $Y$ is completely metrizable, then $C_{V}(X, Y)$ is a Baire space.

PROOF. Let $\rho$ be a compatible bounded complete metric on $Y$. Also let

$\left\{W_{n}: n \varepsilon \omega\right\}$ be a sequence of dense open subsets of $C_{\nu}(X, Y)$, and let $W$ be a nonempty open subset of $C_{\nu}(X, Y)$ Choose $d_{I} \varepsilon M(Y), f_{I} \varepsilon C(X, Y)$, and $0<\varepsilon_{I}<I / 2$ so that

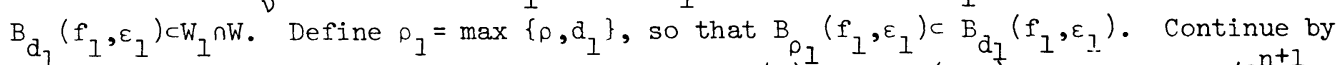
induction so that at the $n+1$ step, choose $d_{n+1} \varepsilon M(Y)^{1}, f_{n+1} \varepsilon C(X, Y)$, and $0<\varepsilon_{n+1}<1 / 2^{n+1}$ such that $B_{d_{n+1}}\left(f_{n+1}, \varepsilon_{n+1}\right) \subset W_{n+1} \cap B_{\rho_{n}}\left(f_{n}, \varepsilon_{n} / 2\right)$; and define $\rho_{n+1}=\max \left\{\rho_{n}, d_{n+1}\right\}$. Now $\left\{f_{n}: n \varepsilon w\right\}^{-}$is a Cauchy sequence in $C_{\rho}(X, Y)$, and therefore converges to some $f \varepsilon C_{\rho}(X, Y)$. Also for each $i \varepsilon \omega, \quad\left\{f_{n}: n \varepsilon \omega\right\}$ will converge to $f$ in $C_{\rho_{i}}(X, Y)$, so that $f$ is in the closure of $B_{\rho_{i}}\left(f_{i,}, \varepsilon_{j} / 2\right)$ in $C_{\rho_{i}}(X, Y)$. Therefore $f \varepsilon B_{\rho_{i}}\left(f_{i}, \varepsilon_{i}\right) \subset B_{d_{i}}\left(f_{i}, \varepsilon_{i}\right) \subset W_{j} \cap W$ for every $i \varepsilon \omega$.

The conclusion of Theorem 1.4 cannot be strengthened to Cech-completeness, which can be seen from Theorem 4.1. Also the hypothesis cannot be changed to Y being compact. For example, if $\mathrm{X}$ is the closed unit interval $\mathrm{I}$ with the usual topology, and $\mathrm{Y}=\mathrm{I}^{2}$ 
with the order topology with respect to lexicographic ordering, then some functions in $\mathrm{C}_{\nu}(\mathrm{X}, \mathrm{Y})$ have open neighborhoods which can be written as countable unions of nowhere dense sets.

2. FINE TOPOLOGIES.

Throughout this section, $(Y, \rho)$ will be a metric space. In this case there is a natural way to generate a topology on $\mathrm{C}(\mathrm{X}, \mathrm{Y})$ which may be even finer than the fine uniformity topology.

For each $f \varepsilon C(X, Y)$ and $\phi \varepsilon C^{+}(X)$, define $B_{\rho}^{+}(f, \phi)=\{\operatorname{g\varepsilon C}(X, Y)$ : for every $x \in X$, $\rho(f(x), g(x))<\phi(x)\}$. Since for each $\phi, \psi \varepsilon C^{+}(X), \max \{\phi, \psi\} \varepsilon C^{+}(X)$, then $\left\{B_{\rho}^{+}(f, \phi): f \varepsilon C(X, Y)\right.$ and $\left.\phi \varepsilon C^{+}(X)\right\}$ is a base for a topology on $C(X, Y)$. This topology is called the fine topology with respect to $\rho$ (Munkres [3], p.285), and will be denoted by $\mathrm{C}_{f_{\rho}}(\mathrm{X}, \mathrm{Y})$. Certainly $\mathrm{C}_{f_{\rho}}(\mathrm{X}, \mathrm{Y})$ is finer than $\mathrm{C}_{\rho}(\mathrm{X}, \mathrm{Y})$, and is in general strictly finer. However, for psuedocompact $X$, they are the same.

PROPOSITION 2.1. If $X$ is pseudocompact, then $C_{f_{\rho}}(X, Y)=C_{\rho}(X, Y)$.

PROOF. Let $\mathrm{f} \mathrm{C}_{f_{\rho}}(X, Y)$, and let $\phi \varepsilon C^{+}(X)$. Since $I / \phi \varepsilon C^{+}(X)$ and since $X$ is psuedocompact, then $I / \phi$ is bounded. So there is a number $M$ with $I / \phi(x)<M$ for all $x \in X$. Therefore $B_{\rho}(f, I / M) \subset B_{\rho}^{+}(f, \phi)$.

From Propositions 1.3 and 2.1 it follows that if $X$ is psuedocompact then $C_{f_{\rho}}(X, Y)=C_{\nu}(X, Y)$. But even if $X$ is not psuedocompact, $C_{f_{\rho}}(X, Y)$ can still be related to $\mathrm{C}_{\nu}(\mathrm{X}, \mathrm{Y})$ as follows.

PROPOSITION 2.2. If $X$ is paracompact, then $C_{\nu_{+}}(X, Y) \leq C_{f_{\rho}}(X, Y)$.

PROOF. Let $\operatorname{d} \varepsilon M(Y)$, let $f \varepsilon C(X, Y)$, and let e $\mathbb{R}^{+}$. For each $x \varepsilon X$, there exists a $\psi(x) \varepsilon \mathbb{R}^{+}$such that $B_{\rho}(f(x), \psi(x)) \subset B_{d}(f(x), e / 2)$. Let $u=\left\{U_{\alpha}: \alpha \varepsilon A\right\}$ be a starrefinement of $\left\{f^{-1}\left(B_{\rho}(f(x), \psi(x) / 2)\right): x \varepsilon X\right\}$. Let $\left\{\phi_{\alpha}: \alpha \varepsilon A\right\}$ be a partition of unity subordinated to $U$. For each $\alpha \varepsilon A$, let $n_{\alpha}$ be the cardinality of $\left\{\beta \varepsilon A: U_{\alpha} \cap U_{\beta} \neq \not\right\}$, let $x_{\alpha} \in X$ be such that $U_{\alpha} \subset f^{-1}\left(B_{\rho}\left(f\left(x_{\alpha}\right), \psi\left(x_{\alpha}\right)\right)\right)$, and let $m_{\alpha}=\min \left\{\psi\left(x_{\beta}\right) / 2: U_{\alpha} \cap U_{\beta} \neq \emptyset\right\}$. Then define $\phi=\Sigma\left\{\left(m_{\alpha} / n_{\alpha}\right) \phi_{\alpha}: \alpha \varepsilon A\right\}$, which is a member of $\mathrm{C}^{+}(\mathrm{X})$.

To establish that $B_{\rho}^{+}(f, \phi) \subset B_{d}(f, e)$, let $g \varepsilon B_{\rho}^{+}(f, \phi)$ and let $x \in X$. Also let

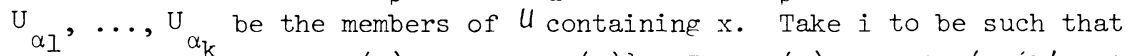

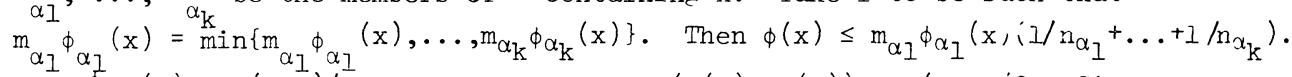
$\leq \mathrm{m}_{\alpha_{i}}{ }^{\phi} \alpha_{i}(x) \leq \psi\left(x_{\alpha_{i}}\right) / 2$. It jollows that $\rho(g(x), f(x))<\psi\left(x_{\alpha_{i}} / / 2\right.$. Since $f(x) \varepsilon_{\rho}\left(f\left(x_{\alpha_{i}}\right), \psi\left(x_{\alpha_{i}}\right) / 2\right)$, then $g(x) \varepsilon_{\rho}\left(f\left(x_{\alpha_{i}}\right), \psi\left(x_{\alpha_{i}}\right)\right) \subset B_{d}\left(f\left(x_{\alpha_{i}}\right), e / 2\right)$. Also $f(x) \in B_{d}\left(f\left(x_{\alpha_{i}}\right), e / 2\right)$, so that $g(x) \varepsilon B_{d}(f(x), e)$.

The inequality in Proposition 2.2 is in general not an equality as indicated in

the comment after Corollary 3.5 .

Whenever $\rho$ is complete then $C_{f_{\rho}}(X, Y)$ is a Baire space ( $\left.[4], p .297\right)$. Instead of giving a proof of this here, a proof will be given in the next section that $C_{f_{\rho}}(X)$ is psuedo-complete, which is a property stronger than being a Baire space.

3. REAL-VALUED FUNCTIONS. For the rest of the paper, $\rho$ will denote the usual metric on $\mathbb{R}$ bounded by 1 ; that is, $\rho(s, t)=\min \{1,|s-t|\}$.

LEMMA 3.1. Let $f, g \in C(X)$ and let $\phi, \psi \varepsilon C^{+}(X)$. Then the closure of $B_{\rho}^{+}(f, \phi)$ in $\mathrm{C}_{f_{\rho}}(\mathrm{X})$ is contained in $\mathrm{B}_{\rho}^{+}(g, \psi)$ if and only if for each $\mathrm{x} \in \mathrm{X}$ the closure of $\mathrm{B}_{\rho}(f(x), \phi(x))$ in $\mathbb{R}$ is contained in $\mathrm{B}_{\rho}(\mathrm{g}(\mathrm{x}), \psi(\mathrm{x}))$. 
PROOF. For the sufficiency, let $h \in B_{\rho}^{+}(f, \phi)$ and let $x \in X$. Suppose $h(x)$ were contained in the complement of the closure of $B_{\rho}(f(x), \phi(x))$; call this set V. Then the set of functions taking $x$ into $V$ would be a neighborhood of $h$ in $C_{f_{\rho}}(x)$ which misses $B_{\rho}^{+}(f, \phi)$. This contradiction shows that $h(x) \varepsilon c l\left(B_{\rho}(f(x), \phi(x))\right)^{f_{\rho}} \subset B_{\rho}(g(x), \phi(x))$.

For necessity, let $x_{0} \varepsilon X$ and let $t \varepsilon c l\left(B_{\rho}\left(f\left(x_{0}\right), \phi\left(x_{0}\right)\right)\right)$ in $\mathbb{R}$. Define $h(x)=f(x)+\left(t-f\left(x_{0}\right)\right) \cdot \phi(x) / \phi\left(x_{0}\right)$ for all $x \in X$. This defines an $h \varepsilon C(x)$ such that $h\left(x_{0}\right)=t$. Also $|h(x)-f(x)| \leq \phi(x)$ for each $x$.

To see that $h \varepsilon c l\left(B_{\rho}^{+}(f, \phi)\right)$ in $C_{f_{\rho}}(x)$, let $B_{\rho}^{+}(h, \xi)$ be a basic neighborhood of $h$. Define $k(x)=h(x)+\operatorname{sign}(f(x)-h(x)) \cdot \min \{\xi(x) / 2,|h(x)-f(x)|\}$, which is an element of $C(x)$. Now $|k(x)-h(x)|=\min \{\xi(x) / 2,|h(x)-f(x)|\} \leq \xi(x) / 2<\xi(x)$. Also if $h(x)>f(x)$, then $|k(x)-f(x)|=|h(x)-f(x)-\min \{\xi(x) / 2, h(x)-f(x)\}|$. If this is positive, then it is equal to $\xi(x) / 2$, and $\xi(x) / 2 \leq|h(x)-f(x)|<\phi(x)$. The same argument shows that if $h(x)<f(x)$, then also $|k(x)-f(x)|<\phi(x)$. Therefore $k \varepsilon B_{\rho}^{+}(f, \phi)$, so that $\mathrm{B}_{\rho}^{+}(h, \xi) \cap \mathrm{B}_{\rho}^{+}(f, \phi) \neq \varnothing$. From this it follows that $\operatorname{hecl}\left(\mathrm{B}_{\rho}^{+}\left(f^{\rho}, \phi\right)\right) \subset \mathrm{B}_{\rho}^{+}(g, \psi)$. But then $\left|t-g\left(x_{0}\right)\right|^{\rho}=\left|h\left(x_{0}\right)-g\left(x_{0}\right)\right|<\psi\left(x_{0}\right)$, so that $t \varepsilon B_{\rho}\left(g\left(x_{0}\right), \psi\left(x_{0}\right)\right)$.

The proof of Lemma 3.1 depends on the metric (and algebraic) structure of the range space. In fact the lemma is not true in general. For example, let $X=I$ and $Y=\left\{(s, \sin (2 \pi / s)) \varepsilon R^{2}: 0<s \leq 2\right\} \cup\{(0,0)\}$ with metric $d$ on $Y$ defined by $d\left(\left(s_{1}, t_{1}\right),\left(s_{2}, t_{2}\right)\right)=\max \left\{\left|s_{1}-s_{2}\right|,\left|t_{1}-t_{2}\right|\right\}$. Also let $y=(2,0)$, let $z=(0,0)$, let $f$ be the constant man taking $X$ to $\mathrm{y}$, and let $\mathrm{g}$ be the constant map taking $\mathrm{X}$ to $\mathrm{z}$. Then $B_{d}(f, 2)=C(X, Y) \backslash\{g\}$. Since $g$ is isolated, $B_{d}(f, 2)$ is closed, so that $c l\left(B_{d}(f, 2)\right) \subset B_{d}(f, 2)$. On the other hand, for any $x \in X, z \varepsilon c l\left(B_{d}(f(x), 2)\right) \backslash B_{d}(f(x), 2)$ in $Y$.

THEOREM 3.2. The space $\mathrm{C}_{f_{\rho_{+}}}(\mathrm{x})$ is psuedo-complete.

PROOF. For each new, let $C_{n}^{+}(X)=\left\{\phi \varepsilon C^{+}(X)\right.$ : for all $\left.x \varepsilon X, \phi(x)<1 / 2^{n}\right\}$, and define $B_{n}=\left\{B_{\rho}^{+}(f, \phi): f \varepsilon C(X)\right.$ and $\left.\phi \varepsilon C_{n}^{+}(X)\right\}$. Each $B_{n}$ is a base for $C_{f_{0}}(X)$. It remains to show that if $B_{n} \varepsilon B_{n}$ for each $n$ with $c l\left(B_{n}\right) \subset B_{n}$ then $n\left\{B_{n}: n \varepsilon \omega\right\} \neq \emptyset$. If each $B_{n}=B_{\rho}^{+}\left(f_{n}, \phi_{n}\right)$, then by Lemma 3.1, for each $n \varepsilon w$ and each $x \varepsilon X$, $c I\left(B_{\rho}\left(f_{n+1}(x), \phi_{n+1}(x)\right)\right) \subset B_{\rho}\left(f_{n}(x), \phi_{n}(x)\right)$. Since each $\phi_{n}(x)<1 / 2^{n}$, then $n\left\{B_{\rho}\left(f_{n}(x), \phi_{n}(x)\right): n \varepsilon \omega\right\}=\{f(x)\}$ for some $f(x) \varepsilon \mathbb{R}$. This defines the function $f$, which is the uniform limit of $\left\{f_{n}: n \varepsilon w\right\}$, and is hence continuous. Clearly $f \varepsilon n\left\{B_{n}: n \varepsilon \omega\right\}$, as desired.

The algebraic structure on $\mathbb{R}$ induces an algebraic structure on $\mathrm{C}(\mathrm{X})$. This structure interacts well with some topologies on $C(X)$. For example, $C_{k}(X)$ and $C_{n}(X)$ are always locally convex linear topological spaces. On the other hand, $\mathrm{C}_{\rho}(\mathrm{X})$ is only a topological group under addition while the scalar multiplication operation is not continuous for non-compact $X$. The space $C_{f_{\rho}}(x)$ behaves much like $C_{\rho}(X)$ in this regard. It is straightforward to show that $C_{f_{\rho}}(X)$ is a topological group under addition. As a result, $\mathrm{C}_{f_{\rho}}(\mathrm{X})$ is homogeneous; and for many arguments it suffices to consider only basic neighborhoods of the zero function, $f_{0}$.

The next result establishes when $f_{0}$ has a countable base. It is stated for $(\mathbb{R}, 0)$, but it is also true for any metric snace containing a nontrivial path.

PROPOSITION 3.3. If $X$ is normal and $f_{0}$ has a countable base in $C_{f_{\rho}}(X)$, then $X$ is countably compact. 
PROOF. Suppose $X$ is not countably compact. Then X contains a countable closed discrete set, $\left\{x_{n}: n \varepsilon \omega\right\}$. Let $\left\{\phi_{n}: n \varepsilon \omega\right\}$ be any sequence in $C^{+}(X)$. The goal is to show that $\left\{B_{\rho}^{+}\left(f_{0}, \phi_{n}\right)\right.$ : new\} cannot be a base at $f_{0}$. For each $n$, let $e_{n} \varepsilon \mathbb{R}^{+}$be such that interval $\left[0, e_{n}\right]$ is contained in $B_{\rho}\left(0, \phi_{n}\left(x_{n}\right) / 2\right)$. Since $X$ is normal, there exists a $\phi \varepsilon \mathrm{C}^{+}(\mathrm{X})$ such that $\phi\left(\mathrm{x}_{\mathrm{n}}\right)=\mathrm{e}_{\mathrm{n}}$ for every $\mathrm{n}$.

It remains to show that $B_{\rho}^{+}\left(f_{0}, \phi_{n}\right) \notin B_{\rho}^{+}(f, \phi)$ for each $n$. Fix $n \varepsilon \omega$, and let $U$ be a neighborhood of $x_{n}$ such that $\phi_{n}(U) \subset\left(\phi_{n}\left(x_{n}\right) / 2, \infty\right)$. Because $X$ is a Tychonoff space, there exists an $f \varepsilon C(X)$ such that $f\left(x_{n}\right)=e_{n}, f(X \backslash U)=\{0\}$, and $f(U) \subset\left[0, e_{n}\right]$. Clearly $f \notin B_{\rho}^{+}\left(f_{0}, \phi\right)$ since $f\left(x_{n}\right)=e_{n}=\phi\left(x_{n}\right)$. To see that $f \varepsilon B_{\rho}^{+}\left(f_{0}, \phi_{n}\right)$, let $x \in X$. If $x \notin U$, then $\rho\left(f(x), f_{0}(x)\right)=0$. If $x \varepsilon U$, then $f(x) \varepsilon\left[0, e_{n}\right] \subset B_{\rho}\left(0, \phi_{n}\left(x_{n}\right) / 2\right)$. Also $\phi_{n}(x)>\phi_{n}\left(x_{n}\right) / 2$, so that $\rho\left(f(x), f_{0}(x)\right)<\phi_{n}(x)$.

Therefore for a normal space $X, C_{f_{\rho}}(X)$ is first countable if and only if it is already equal to the metrizable space $\mathrm{C}_{\rho}(\mathrm{X})$.

The situation is little different for $C_{v}(x)$. To begin with, $C_{v}(x)$ is in general not homogeneous, as the next proposition shows.

PROPOSITION 3.4. An element of $\mathrm{C}_{v}(\mathrm{X})$ has a countable base if and only if it is a bounded function.

PROOF. First suppose $f$ is an unbounded function in $C(X)$. Without loss of generality, suppose there is a sequence $\left\{x_{n}: n \varepsilon \omega\right\}$ in $X$ such that $f\left(x_{n}\right)=n$ for each new. Let $\left\{u_{n}[f]: n \varepsilon \omega\right\}$ be any sequence of basic neighborhoods of $f$ in $C_{v}(X)$.

For each $n \varepsilon w$, let $V_{n} \varepsilon U_{n}$ with $n \varepsilon V_{n}$, and let $I_{n}$ be a closed interval containing $\mathrm{n}$ in its interior and contained in $\mathrm{V}_{\mathrm{n}} \mathrm{n}(\mathrm{n}-1 / 2, \mathrm{n}+1 / 2)$. Also let $t_{\mathrm{n}}$ be a point of the interior of $I_{n}$ different than $n$, and $W_{n}$ be an open subset of the interior of $I_{n}$ which contains $n$ but not $t_{n}$. Then let $W=\mathbb{R} \backslash w$, and define $u=\{W\} \cap\left\{W_{n}: n \varepsilon \omega\right\}$.

To see that each $\hat{u}_{n}[f]$ is not contained in $\hat{U}[f]$, let $\phi_{n}: I_{n} \rightarrow I_{n}$ be a homeomorphism which fixes the endpoints of $I_{n}$ and moves $n$ to $t_{n}$. Then define $\phi \otimes C(\mathbb{R})$ by $\phi(s)=\phi_{n}(s)$ if $s \varepsilon I_{n}$ and $\phi(s)=s$ otherwise. It follows that $\phi \circ f \varepsilon \hat{U}_{n}[f] \backslash \hat{U}[f]$. Therefore $\left\{\hat{u}_{n}[f]\right.$ : $\left.n \varepsilon \omega\right\}$ cannot be a base at $f$.

For the converse, suppose that $f$ is a bounded function in $C(X)$. The goal will be to show that $\left\{B_{\rho}(f, I / n): n \varepsilon w\right\}$ is a base at $f$ in $C_{v}(X)$. Let $d \varepsilon M(\mathbb{R})$ and let $e=0$. Also let $M \varepsilon \omega$ such that $f(X)$ is contained in the interval $[-M, M]$. For each $t \varepsilon[-M, M]$, there exists an $e_{t} \varepsilon \mathbb{R}^{+}$such that $B_{\rho}\left(t, e_{t}\right) \subset B_{d}(t, e / 3)$. There exist $t_{1}, \ldots, t_{m} \varepsilon[-M, M]$ such that $[-M, M] \subset B_{\rho}\left(t_{I}, e_{t_{I}} / 2\right) \cap \ldots \cap B_{\rho}\left(t_{m}, e_{t_{m}} / 2\right)$. Take new with $I / n \leq \min \left\{e_{t_{I}} / 2, \ldots\right.$, $\left.e_{t_{m}} / 2\right\}$.

To see that $B_{\rho}(f, I / n) \subset B_{d}(f, e)$, let $g \in B_{\rho}(f, l / n)$ and let $x \in X$. There is some $k$ with $f(x) \varepsilon B_{\rho}\left(t_{k}, e_{t_{k}} / 2\right)$. Now $\rho(g(x), f(x))<l / n \leq e_{t_{k}} / 2$ and $\rho\left(f(x), t_{k}\right)<e_{t_{k}} / 2$, so that $\rho\left(g(x), t_{k}\right)<e_{t_{k}}$. Therefore $g(x) \varepsilon B_{\rho}\left(t_{k}, e_{t_{k}}\right) \subset B_{d}\left(t_{k}, e / 3\right)$, and similarly $f(x) \varepsilon B_{d}\left(t_{k}, e / 3\right)$. Hence $d(g(x), f(x))<2 e / 3$, so that $d(g, f) \leq 2 e / 3<e$.

COROLIARY 3.5. If $\mathrm{C}_{v}(\mathrm{X})$ is first countable, then $\mathrm{X}$ is psuedocompact.

It follows from Proposition 3.4 that whenever $X$ is not psuedocompact then

$C_{v}(X)$ is not homogeneous, and is thus not a topological group under addition. Therefore $C_{\nu}(X)$ and $C_{f_{0}}(X)$ are different whenever $X$ is not psuedocompact. 
4. COUNTABILITY PROPERTIES

The spaces $q_{\nu}(X)$ and $C_{f_{\rho}}(X)$ have a property which is useful for studying countability properties. That property is submetrizability; i.e., these topologies contain weaker metrizable topologies. Certain results follow immediately. For example, singleton sets in $C_{v}(X)$ and $C_{f_{\rho}}(X)$ are $G_{\delta}$-sets. Also the concepts of compactness, countable compactness, anā sequential compactness are equivalent for subsets of these spaces.

There is a concept which is weaker than first countability that will be useful to consider. A space is of point countable type if every point is contained in a compact set which has a countable base. Every Cech-complete space has this property. Also a space which is of point countable type and in which singleton sets are $\mathrm{G}_{\delta}$-sets is first countable. As a result, a number of properties are equivalent for the fine and fine uniform topologies. The proof of the following theorem then follows from Proposition 1.3 and Corollary 3.5.

THEOREM 4.1. If $\mathrm{C}(\mathrm{X})$ has the fine uniform topology (or the fine topology for normal $X)$, then the following are equivalent.

(a) $\mathrm{C}(\mathrm{X})$ is first countable.

(b) $\mathrm{C}(\mathrm{X})$ is of point countable type.

(c) $\mathrm{C}(\mathrm{X})$ is Cech-complete

(d) $C(X)$ is metrizable.

(e) $\mathrm{C}(\mathrm{X})$ is completely metrizable.

(f) $C(X)=C_{\rho}(X)$.

(g) All compatible uniformities on $\mathbb{R}$ induce the same topology on $C(X)$.

(h) $\mathrm{X}$ is pseudocompact.

Theorem 4.1 is also true with $\mathbb{R}$ replaced by any complete metric space which contains a closed ray; i.e., a closed copy of the interval $[0, \infty)$.

THEOREM 4.2. If $\mathrm{C}(\mathrm{X})$ has the fine uniform topology (or the fine topology), then the following are equivalent.

(a) $C(X)$ is separable.

(b) $C(X)$ has the countable chain condition.

(c) $C(X)$ is Iindelof.

(d) $C(X)$ has a countable network.

(e) $C(X)$ is second countable.

(f) $C(X)$ is separable and completely metrizable.

(g) $C_{\rho}(X)$ is separable.

(h) $\mathrm{X}$ is compact and metrizable.

PROOF. Since $C_{\rho}(X) \leq C(X)$, each of $(a)$ through $(f)$ implies $(g)$. That $(g)$ implies $(h)$ is well-known. Finally, if $(h)$ is true, then $C(X)=C_{\rho}(X)$.

These same arguments can be extended to generalize Theorem 4.2. In particular, this theorem will be true if $\mathbb{R}$ is replaced by a separable complete metric space which contains a nontrivial path. 


\section{REFERENCES}

1. EKLUND, A. The fine topology and other topologies on $C(X, Y)$, Dissertation, Virginia Polytechnic Institute and State University, 1978.

2. MCCOY, R.A. Characterization of psuedocompactness by the topology of uniform convergence on function spaces, J. Austral. Math. Soc. 26 (1978), $251-256$.

3. MUNKRES, J.R. Topology, Prentice-Hall, Englewood Cliffs, New Jersey, 1975.

4. TORUNCZYK, H. Characterizing Hilbert space topology, Fund. Math. 111 (1981), $248-262$

5. WILIARD, S. General Topology, Addison-Wesley, Reading Massachusetts, 1968. 


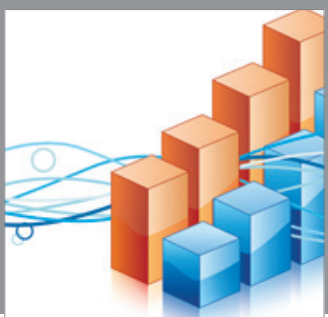

Advances in

Operations Research

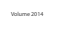

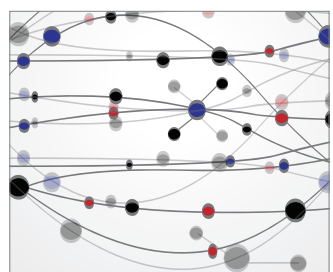

\section{The Scientific} World Journal
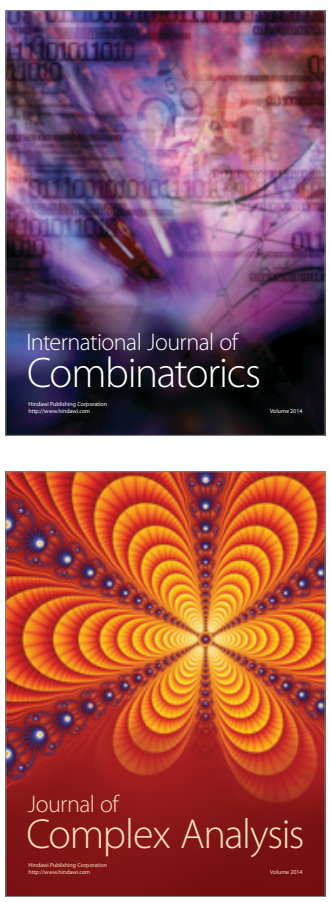

International Journal of

Mathematics and

Mathematical

Sciences
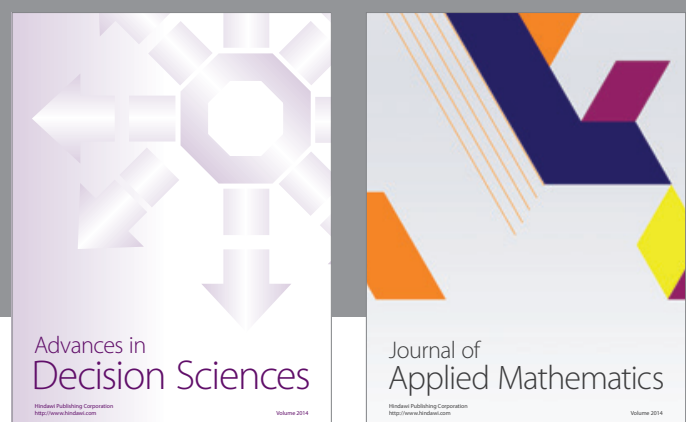

Journal of

Applied Mathematics
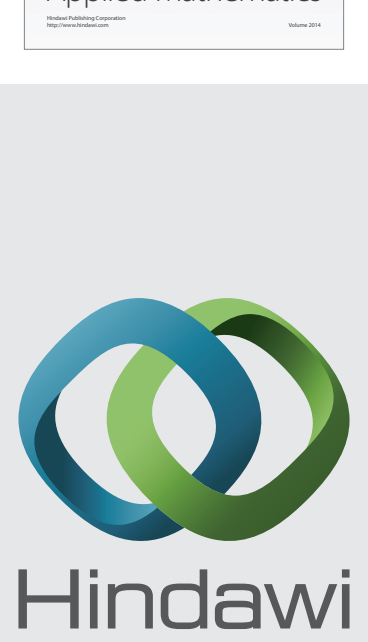

Submit your manuscripts at http://www.hindawi.com
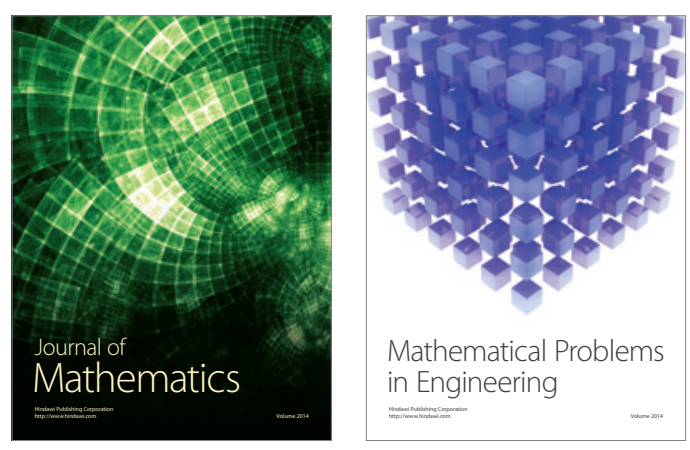

Mathematical Problems in Engineering
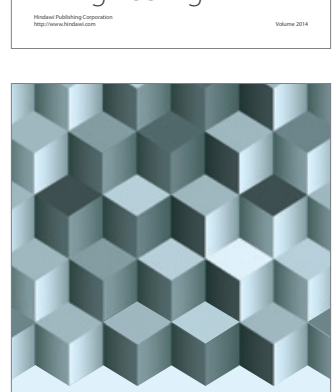

Journal of

Function Spaces
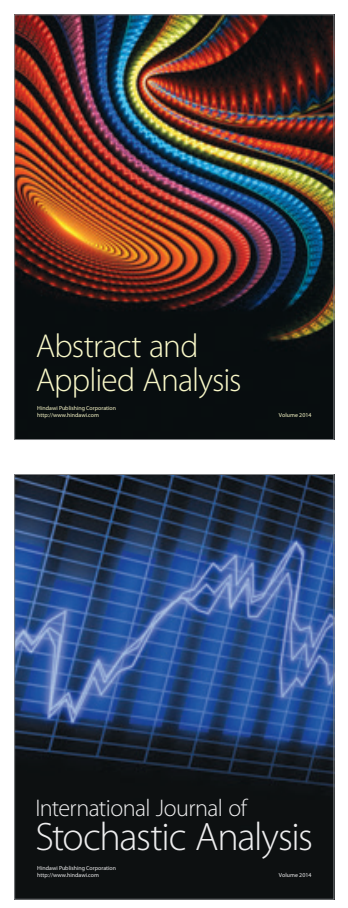

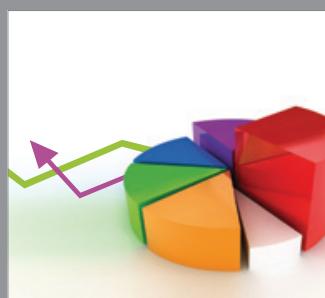

ournal of

Probability and Statistics

Promensencen
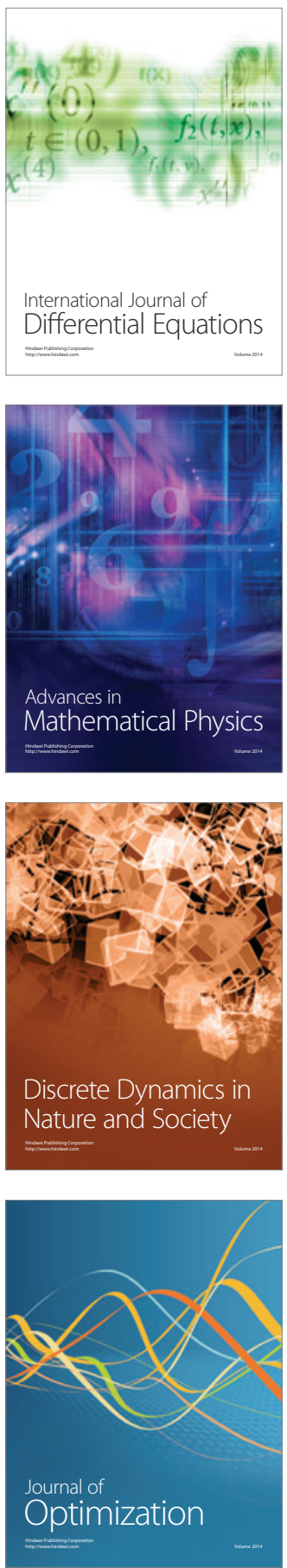